\title{
Bureau d'expertises extrajudiciaires de la FMH: rapport annuel 2019
}

\author{
Valérie Rothhardta, Caroline Hartmann ${ }^{b}$ \\ ${ }^{a}$ avocate, co-responsable du Bureau d'expertises; ${ }^{b}$ avocate, Dre en droit, co-responsable du Bureau d'expertises
}

\section{Rôle du Bureau d'expertises}

Le Bureau d'expertises extrajudiciaires de la FMH mandate un ou plusieurs experts, à la demande d'un patient ayant été traité en Suisse, pour déterminer si, dans le cas concret, un médecin exerçant en pratique privée ou à l'hôpital a violé son devoir de diligence ou si l'hôpital a commis une faute liée à l'organisation. Les experts sont proposés ou confirmés par la société de discipline médicale concernée, ce qui permet de trouver des experts indépendants et compétents. Les assurances de responsabilité civile (membres de l'Association Suisse d'Assurances [ASA], ci-après assurances RC) des médecins ou des hôpitaux prennent en charge les honoraires des experts. Le patient doit s'acquitter d'une taxe administrative de 1000 CHF plus TVA pour obtenir une expertise écrite ou orale.

Ainsi, le Bureau d'expertises est un instrument utile et efficace pour les patients et pour les médecins: il permet aux patients de faire éclaircir la question de savoir s'ils ont été victimes d'une faute médicale à des coûts peu élevés et il donne une base fiable aux médecins respectivement à leur assurance $\mathrm{RC}$ pour régler le litige de manière extrajudiciaire.

En 2019, le Bureau d'expertises extrajudiciaires de la FMH a procédé à l'établissement de 45 expertises. Les experts ont conclu à une ou plusieurs violations du devoir de diligence dans 15 cas et n'ont constaté aucune violation du devoir de diligence dans 30 autres cas.

\section{Procédure}

Le Bureau d'expertises de la FMH n'est pas compétent pour tous les litiges. Pour qu'il organise une expertise, il faut notamment que le patient ait subi un dommage à sa santé et que les parties ne soient pas parvenues à s'entendre à l'amiable. Le Bureau d'expertises de la FMH organisant des expertises extrajudiciaires, il ne faut pas qu'un tribunal se soit déjà prononcé sur la question de la violation du devoir de diligence ou de la faute liée à l'organisation, ni qu'une procédure soit en cours à ce sujet.

En 2019, le Bureau d'expertises a totalement révisé son règlement; le nouveau règlement est entré en vigueur le $1^{\text {er }}$ octobre 2019 (cf. ci-dessous page 731). Les grandes étapes de la procédure sont présentées à la page suivante (figure 1).

\section{Classification en cas d'expertise multidisciplinaire}

La classification tient compte des violations du devoir de diligence constatées dans chaque discipline. Exemple: choix d'un expert principal spécialiste en gynécologie et d'un co-expert spécialiste en anesthésiologie.

- Violation du devoir de diligence reconnue seulement en gynécologie: classement exclusivement sous gynécologie.

- Violation du devoir de diligence reconnue seulement en anesthésiologie: classement exclusivement sous anesthésiologie.

- Violation du devoir de diligence reconnue dans ces deux disciplines: classement sous gynécologie et anesthésiologie.

\section{Analyse de la statistique et comparaison avec les années précédentes}

Expertises rendues en 2019: 45

- dont traitements prodigués par des médecins en cabinet privé: 26 (25,8\%)

- dont traitements exclusivement hospitaliers: 18 $(61,3 \%)$

- dont traitements mixtes: 2 (12,9\%)

- dont équipes multidisciplinaires d'experts: 3

La moyenne des expertises de ces cinq dernières années (2014-2018) s'élève à 50. Le nombre de 45 se situe donc légèrement en-dessous. 
Pourcentage de violations du devoir de diligence reconnues en 2019: 33,3\%

- en 2018: $45,2 \%$

- lors des dix années précédentes (2009-2018): entre 31,5 et $50,6 \%$

- lors des cinq années précédentes (2014-2018): entre 31,5 et $45,2 \%$

Le pourcentage de violations du devoir de diligence reconnues en 2019 se situe dans la fourchette inférieure des années précédentes.

Nombre de décisions de non-entrée en matière rendues en 2019: 13

- en 2018: 15

- en moyenne lors des cinq dernières années (20142018): 10

\section{Limites quant à l'interprétation de la statistique}

\section{Pas de représentativité au niveau suisse}

Ces chiffres reflètent uniquement l'activité du Bureau d'expertises de la FMH durant l'année 2019. Or, notre Bureau n'a pas le monopole en matière d'établissement d'expertises, les patients organisent régulièrement des expertises privées et les hôpitaux répondent euxmêmes à plusieurs reproches de violation du devoir de diligence par année. Au vu du petit nombre de données à disposition et du manque de valeurs comparatives, ces statistiques ne sauraient être prises comme base de calcul pour déterminer le nombre de violations des règles de l'art annuelles en Suisse ou quelles disciplines sont les plus touchées.

Dépôt de la demande d'expertise - taxe administrative de Fr. 300.- à charge du demandeur

- Demande déposée par le patient ou son représentant juridique

- Ou par la personne autorisée à le représenter, ses héritiers ou son concubin

- La demande doit être motivée et documentée

Nomination d'expert/s et procédure de récusation

- Soit proposition d'expert/s par la société de discipline médicale et procédure de récusation

- Soit proposition d'expert/s par les parties et confirmation par la société de discipline médicale

- L'implication de la société de discipline garantit la compétence et la neutralité de/s expert/s

Procédure écrite

- Le projet d'expertise est transmis au Bureau d'expertises

- Contrôle de qualité par un juriste

- Garantie d'une expertise complète, claire et cohérente

Octroi du mandat - taxe administrative de Fr. 700.- à charge du demandeur

- L'octroi du mandat à l'expert est effectué par le Bureau d'expertises

- Délai de 4 mois pour la rédaction du projet d'expertise ou la préparation de l'expertise conjointe de la FMH
Eventuellement : dépôt de questions complémentaires écrites

- Dans les deux mois dès la remise de l'expertise

- Le Bureau d'expertises décide quelle/s question/s sont transmises à l'expert

- Exclut une demande d'explication orale
Procédure orale (expertise conjointe de la FMH)

- Réalisation de l'expertise autour d'une table ronde

- Procès-verbal approuvé par les participants

- Projet pilote jusqu'au 31 décembre 2021

Figure 1:Déroulement de la procédure. 
Statistiques du Bureau d'expertises extrajudiciaires

Tableau 1: Statistique détaillée, année 2019

\begin{tabular}{|l|l|l|l|l|}
\hline & $\begin{array}{l}\text { Expertises } \\
\text { établies }\end{array}$ & $\begin{array}{l}\text { Violation des } \\
\text { règles de l'art } \\
\text { avérée }\end{array}$ & $\begin{array}{l}\text { Violation des } \\
\text { règles de l'art } \\
\text { niée }\end{array}$ & $\begin{array}{l}\text { Violation des } \\
\text { règles de l'art } \\
\text { indéterminée }\end{array}$ \\
\hline Suisse alémanique & 22 & 9 & 13 & 0 \\
Tessin & 2 & 0 & 2 & 0 \\
\hline Suisse romande & 21 & 6 & 15 & 0 \\
\hline Toute la Suisse & $\begin{array}{l}45 \\
(100 \%)\end{array}$ & $\begin{array}{l}15 \\
(33.3 \%)\end{array}$ & $\begin{array}{l}30 \\
(66.7 \%)\end{array}$ & $\begin{array}{l}0 \\
(0.0 \%)\end{array}$
\end{tabular}

Tableau 2: Lien de causalité, année 2019

\begin{tabular}{|l|l|l|l|l|}
\hline & $\begin{array}{l}\text { Violation des } \\
\text { règles de l'art } \\
\text { avérée }\end{array}$ & $\begin{array}{l}\text { Causalité } \\
\text { confirmée }\end{array}$ & Causalité niée & $\begin{array}{l}\text { Causalité } \\
\text { indéterminée }\end{array}$ \\
\hline $\begin{array}{l}\text { Suisse alémanique } \\
\text { Tessin }\end{array}$ & 9 & 4 & 5 & 0 \\
\hline $\begin{array}{l}\text { Suisse romande } \\
\text { Toute la Suisse }\end{array}$ & 0 & 0 & 0 & 0 \\
\hline & 15 & 4 & 2 & 0 \\
\hline & $(100 \%)$ & 8 & 7 & 0 \\
\hline
\end{tabular}

Tableau 3: Non-entrées en matière, année 2019

\begin{tabular}{|l|l|}
\hline & $\begin{array}{l}\text { Non-entrée } \\
\text { en matière }\end{array}$ \\
\hline $\begin{array}{l}\text { Suisse alémanique } \\
\text { Tessin } \\
\text { Suisse romande }\end{array}$ & 4 \\
\hline Toute la Suisse & 9 \\
\hline
\end{tabular}

Tableau 4: Statistique globale, années 1982-2019

\begin{tabular}{|l|l|l|l|l|}
\hline Toute la Suisse & $\begin{array}{l}\text { Expertises } \\
\text { établies }\end{array}$ & $\begin{array}{l}\text { Violation des } \\
\text { règles de l'art } \\
\text { avérée }\end{array}$ & $\begin{array}{l}\text { Violation des } \\
\text { règles de l'art } \\
\text { niée }\end{array}$ & $\begin{array}{l}\text { Violation des } \\
\text { règles de l'art } \\
\text { indéterminée }\end{array}$ \\
\hline $1982-2019$ & $\begin{array}{l}3831 \\
(100 \%)\end{array}$ & $\begin{array}{l}1312 \\
(34.2 \%)\end{array}$ & $\begin{array}{l}2420 \\
(63.2 \%)\end{array}$ & $\begin{array}{l}99 \\
(2.6 \%)\end{array}$ \\
\hline $2010-2019$ & $\begin{array}{l}598 \\
(100 \%)\end{array}$ & 247 & 345 & 6 \\
& $(41.3 \%)$ & $(57.7 \%)$ & $(1 \%)$
\end{tabular}

Reflet partiel du travail fourni par le Bureau d'expertises

La statistique reflète seulement les résultats relatifs aux expertises rendues - soit 45 en 2019 - et ne montre pas le grand travail administratif fourni par notre Bureau: ce dernier analyse les nouvelles demandes qui lui ont été adressées sous l'angle du règlement et requiert les éventuels documents manquants. En 2019, 98 nouvelles demandes nous ont été adressées. De celles-ci, 46 ont été envoyées au délégué de la société de discipline médicale concernée. Les autres demandes sont en cours de traitement, notamment en raison du fait que les parties n'ont pas encore fourni tous les documents nécessaires. Par ailleurs, même si les problèmes exposés n'entrent pas dans son domaine de compétence, le Bureau d'expertises s'efforce de conseiller au mieux les patients qui s'adressent à lui.

\section{Lien de causalité entre la faute et le dommage à la santé}

Si une violation des règles de l'art ou du devoir d'information respectivement une faute liée à l'organisation a été constatée, l'expert doit déterminer si cette violation est à l'origine du dommage à la santé évoqué par le patient.

Pour cela, il doit déterminer quel aurait été l'état de santé du patient sans la violation du devoir de diligence constatée. Si, malgré la violation du devoir de diligence, le patient avait eu le même état de santé, celle-ci n'est pas causale. L'expert se détermine sur le lien de causalité uniquement d'un point de vue médical et non juridique.

En 2019, les experts ont reconnu un lien de causalité dans 8 cas sur les 15 où une violation des règles de l'art a été constatée. Dans 7 cas, le lien de causalité a été nié.

\section{Information médicale au patient et communication entre médecin et patient}

La question de savoir si l'information médicale donnée au patient était suffisante ne peut pas à elle seule faire l'objet d'une expertise de la FMH. Elle peut toutefois être abordée parallèlement à la violation du devoir de diligence ou à la faute liée à l'organisation supposée. Une information claire, complète et bien documentée revêt une importance capitale. D'une part, pour que le patient comprenne les implications du traitement et puisse prendre des décisions en toute connaissance de cause. D'autre part, pour que le médecin soit en mesure de prouver comment il a informé le patient et qu'il a respecté son obligation légale.

En 2019, les experts ont constaté une information insuffisante dans six cas dans lesquels aucune violation des règles de l'art n'a été commise. La causalité a été admise dans un seul de ces cas.

\section{Assurance-qualité}

Rendre des expertises de qualité est essentiel. Les démarches suivantes garantissent une bonne qualité:

- Les sociétés de discipline médicale nomment un ou plusieurs experts pour chaque cas particulier. Lorsque le cas l'exige, une équipe pluridisciplinaire d'experts est constituée. Par ce procédé, une évaluation du cas par des experts neutres et compétents est assurée. Le mandat est octroyé à l'expert/aux experts au terme d'une procédure de récusation. En principe, l'expertise est rendue dans la langue du patient (français, allemand ou italien).

- Les experts travaillent avec un schéma utilisé depuis de nombreuses années. Cela les aide à structurer l'expertise et à aborder tous les points déterminants. Ainsi, les parties disposent d'une expertise leur permettant de régler au mieux le litige. 
- Le Service juridique de la FMH relit le projet d'expertise. Cet instrument d'assurance-qualité apporte le regard d'un non-médecin et soutient les experts dans la rédaction de l'expertise.

\section{Révision du règlement}

En 2019, le Bureau d'expertises a terminé la révision de son règlement, entamée en 2018. Il dispose ainsi d'un règlement entièrement révisé depuis le $1^{\mathrm{er}}$ octobre 2019. Les nouveautés les plus importantes sont les suivantes:

- Les parties peuvent s'entendre pour procéder à une expertise conjointe de la FMH. Il s'agit d'une procédure orale, où l'expert explique les questions médicales aux parties autour d'une table ronde.
- Lorsqu'une expertise écrite a été rendue, les parties peuvent en demander une explication orale. Il s'agira alors d'éclaircir certains points, sans que de nouvelles questions soient posées à l'expert.

- Les parties peuvent proposer un ou plusieurs experts d'un commun accord.

- Elles peuvent - également d'un commun accord convenir de questions à poser à l'expert.

- L'expert dispose à présent d'un délai de quatre mois pour remettre le projet d'expertise au juriste du Bureau d'expertises.

- Enfin, le demandeur doit s'acquitter d'une taxe administrative non remboursable de 300 CHF (plus TVA) avant que sa demande ne soit examinée par le Bureau d'expertises. Si une expertise est organisée

\section{Statistiques du Bureau d'expertises extrajudiciaires}

Figure 2: Tableau 5: Résultats par spécialité, année 2019 et années 1992-2019.

\begin{tabular}{|c|c|c|c|c|c|c|c|c|}
\hline & \multicolumn{2}{|c|}{$\begin{array}{l}\text { Expertises } \\
\text { établies }\end{array}$} & \multicolumn{2}{|c|}{$\begin{array}{l}\text { Violation des } \\
\text { règles de l'art } \\
\text { avérée }\end{array}$} & \multicolumn{2}{|c|}{$\begin{array}{c}\text { Violation des } \\
\text { règles de l'art } \\
\text { niée }\end{array}$} & \multicolumn{2}{|c|}{$\begin{array}{l}\text { Violation des } \\
\text { règles de l'art } \\
\text { indéterminée }\end{array}$} \\
\hline & 2019 & $\begin{array}{l}1982- \\
2019\end{array}$ & 2019 & $\begin{array}{l}1982- \\
2019\end{array}$ & 2019 & $\begin{array}{c}1982- \\
2019\end{array}$ & 2019 & $\begin{array}{l}1982- \\
2019\end{array}$ \\
\hline Médecine interne générale & 3 & 500 & 1 & 180 & 2 & 306 & 0 & 14 \\
\hline Anesthésiologie & 1 & 127 & 0 & 41 & 1 & 83 & 0 & 3 \\
\hline Chirurgie & 0 & 868 & 0 & 309 & 0 & 532 & 0 & 27 \\
\hline Dermatologie & 0 & 31 & 0 & 9 & 0 & 20 & 0 & 2 \\
\hline Gastro-entérologie & 2 & 19 & 2 & 6 & 0 & 13 & 0 & 0 \\
\hline Gynécologie et obstétrique & 4 & 487 & 1 & 185 & 3 & 294 & 0 & 8 \\
\hline Chirurgie de la main & 1 & 68 & 1 & 24 & 0 & 42 & 0 & 2 \\
\hline $\begin{array}{l}\text { Chirurgie cardiaque et } \\
\text { vasculaire thoracique }\end{array}$ & 0 & 28 & 0 & 9 & 0 & 18 & 0 & 1 \\
\hline Cardiologie & 1 & 27 & 0 & 12 & 1 & 14 & 0 & 1 \\
\hline Chirurgie maxillo-faciale & 0 & 27 & 0 & 3 & 0 & 24 & 0 & 0 \\
\hline Chirurgie pédiatrique & 0 & 15 & 0 & 5 & 0 & 10 & 0 & 0 \\
\hline Psychiatrie pédiatrique & 0 & 1 & 0 & 0 & 0 & 1 & 0 & 0 \\
\hline Néphrologie & 0 & 2 & 0 & 0 & 0 & 2 & 0 & 0 \\
\hline Neurochirurgie & 0 & 112 & 0 & 35 & 0 & 75 & 0 & 2 \\
\hline Neurologie & 0 & 29 & 0 & 8 & 0 & 20 & 0 & 1 \\
\hline Oncologie & 1 & 10 & 0 & 4 & 1 & 6 & 0 & 0 \\
\hline Ophtalmologie & 4 & 155 & 2 & 47 & 2 & 102 & 0 & 6 \\
\hline Chirurgie orthopédique & 22 & 784 & 6 & 292 & 16 & 477 & 0 & 15 \\
\hline Oto-rhino-laryngologie ORL & 1 & 126 & 0 & 31 & 1 & 91 & 0 & 4 \\
\hline Pédiatrie & 1 & 74 & 1 & 31 & 0 & 40 & 0 & 3 \\
\hline Pathologie & 0 & 6 & 0 & 4 & 0 & 2 & 0 & 0 \\
\hline Pharmacologie & 0 & 2 & 0 & 2 & 0 & 0 & 0 & 0 \\
\hline $\begin{array}{l}\text { Médecine physique et } \\
\text { réadaptation }\end{array}$ & 0 & 13 & 0 & 3 & 0 & 9 & 0 & 1 \\
\hline $\begin{array}{l}\text { Chirurgie plastique, } \\
\text { reconstructive et esthétique }\end{array}$ & 0 & 133 & 0 & 28 & 0 & 103 & 0 & 2 \\
\hline Pneumologie & 0 & 3 & 0 & 2 & 0 & 1 & 0 & 0 \\
\hline Psychiatrie & 2 & 21 & 1 & 8 & 1 & 13 & 0 & 0 \\
\hline Radiologie & 0 & 59 & 0 & 15 & 0 & 40 & 0 & 4 \\
\hline Radio-oncologie & 0 & 1 & 0 & 1 & 0 & 0 & 0 & 0 \\
\hline Rhumatologie & 0 & 18 & 0 & 6 & 0 & 12 & 0 & 0 \\
\hline Chirurgie thoracique & 1 & 1 & 0 & 0 & 1 & 1 & 0 & 0 \\
\hline Urologie & 1 & 84 & 0 & 12 & 1 & 69 & 0 & 3 \\
\hline Total 2019, 1982 - 2019 & 45 & 3831 & 15 & 1312 & 30 & 2420 & 0 & 99 \\
\hline
\end{tabular}


(par écrit ou par oral), une taxe supplémentaire de 700 CHF (plus TVA) lui est demandée. L'explication orale d'une expertise écrite coûte 300 CHF supplémentaires (plus TVA).

Vous trouverez de plus amples informations à ce sujet dans le Bulletin des médecins suisses n 38 (2019), pp. 1252 et 1253.

\section{Nouvelle disposition du Code de déontologie}

Dans le cadre de la révision du règlement du Bureau d'expertises, la Chambre médicale a introduit, par décision du 9 mai 2019, une nouvelle clause dans le Code de déontologie de la FMH, qui est entrée en vigueur le $1^{\mathrm{er}}$ avril 2020. Elle dispose que les membres de la FMH sont tenus de prendre en charge les honoraires des experts du Bureau d'expertises lorsque leur assurance RC professionnelle n'est pas membre de l'Association Suisse d'Assurances (ASA) et qu'elle n'accorde pas de garantie de paiement. Si l'assurance RC est membre de l'ASA, elle paie les honoraires des experts sur la base d'un gentlemen's agreement passé en 1982 entre la FMH et l'ASA indépendamment du résultat de l'expertise et de l'avis de l'assurance RC quant à l'utilité d'une telle expertise.

Pour plus de précisions, voir l'article paru dans le Bulletin des médecins suisses nº 39 (2019), pp. 1292 et 1293.

\section{Conférences}

Les avocates du Bureau d'expertises donnent régulièrement des conférences pour former les médecins à l'expertise médicale ou aborder la question de la responsabilité civile du médecin. En 2019, elles sont intervenues au forum d'anesthésiologie à Lucerne, auprès de la Swiss Insurance Medicine (SIM) à Bâle et lors d'un séminaire HAVE pour avocats et juristes à Lucerne.

\section{Durée de la procédure}

En 2019, les procédures d'expertise ont duré en moyenne 16 mois et demi après le dépôt de la demande. Plusieurs cas ont cependant été achevés en moins d'un an.

Bien entendu, cette attente est longue, avant tout pour les patients, qui souhaitent avoir une réponse à leurs questions, et les médecins mis en cause. La longueur de la procédure s'explique, entre autres, pour les raisons suivantes:

- La procédure est réglementée, transparente et implique toutes les parties. Cela prend du temps. Selon les cas, la recherche d'experts compétents dure à elle seule plusieurs mois, en particulier lorsque les experts proposés sont récusés par l'une des parties.

- Souvent, réunir les documents nécessaires à la réalisation de l'expertise est laborieux. De plus, peu de demandes sont complètes dès le début.

- La charge de travail de nombreux experts est telle qu'ils trouvent à peine le temps nécessaire pour effectuer ce genre de mandats supplémentaires dans le délai souhaité; la plupart y sacrifient une partie de leurs moments de loisirs.

- A cela s'ajoute le temps investi par le Service juridique de la FMH pour lire les projets d'expertises, et le cas échéant, le temps dont a besoin l'expert pour compléter son expertise.

- Lorsque plusieurs experts ont été mandatés, chaque étape requiert plus de temps, depuis l'audition et l'examen du patient jusqu'à la rédaction finale de l'expertise.

Le Bureau d'expertises extrajudiciaires ne détient pas le monopole en matière d'établissement d'expertises. S'il accepte d'organiser une expertise, il dirige la procédure conformément à son règlement et traite toutes les parties équitablement.

\section{Formulaire de feed-back: résultats}

Par le biais du formulaire de feed-back, envoyé aux parties six mois après que l'expertise a été rendue, le Bureau d'expertises peut savoir comment ses expertises sont acceptées et utilisées par les parties. En effet, tant les patients que les assurances RC prennent la peine de le compléter et de nous le renvoyer.

Les résultats de l'année 2019 concordent avec ceux des années précédentes, en fonction du résultat de l'expertise:

- Constatation d'une violation du devoir de diligence et/ou du devoir d'information ainsi que du lien de causalité: les assurances RC en acceptent souvent le résultat et les patients sont alors indemnisés. Parfois, le lien de causalité est contesté par l'assurance et les deux parties doivent négocier. Tous les patients ne sont pas satisfaits de l'indemnisation qui leur est offerte. Certains choisissent alors d'intenter une action civile.

- Pas de constatation d'une violation du devoir de diligence et/ou du devoir d'information ou constatation d'une telle violation mais non du lien de causalité: les patients sont alors mécontents du résultat mais ils renoncent très majoritairement à intenter une action ou à demander une expertise privée. 


\section{Conseil scientifique}

Le Conseil scientifique surveille l'activité du Bureau d'expertises extrajudiciaires sur mandat du Comité central de la FMH. Il n'a pas de compétence décisionnelle mais décharge le Comité central de son devoir de surveillance et soutient le Bureau d'expertises en cas de difficultés dans un dossier. Au cours de l'exercice en revue, le Conseil scientifique s'est réuni à deux reprises et a examiné par sondage huit dossiers d'expertise et huit décisions de non-entrée en matière.

Suite à la révision du règlement, le Conseil scientifique comporte un membre supplémentaire pour représenter la SIM. Au mois de novembre 2019, le Conseil scientifique a déjà siégé dans sa nouvelle composition qui se présente comme suit:

- Dr Andreas Rindlisbacher, président, représentant des médecins

- Dr Jürg Knessl, représentant des patients

- Michel Bögli, licencié en droit, représentant des assurances

- Dr Gerhard Ebner, représentant de la SIM

\section{Remerciements}

Le Bureau d'expertises extrajudiciaires de la FMH ne peut fonctionner qu'avec l'aide de nombreuses personnes. Nous remercions les sociétés de discipline médicale et leurs délégués pour leur précieuse collaboration ainsi que les experts pour leur disponibilité et le grand travail qu'ils accomplissent. Merci également aux médecins traitants et aux directions d'hôpitaux qui ont coopéré à la réalisation d'expertises.

\section{Conseil aux patients}

Bureau d'expertises extrajudiciaires de la FMH Case postale 65 CH-300o Berne 15
Avant de déposer votre demande, contactez par téléphone le Bureau d'expertises extrajudiciaires de la FMH.
Ces discussions préalables permettent d'éclaircir nombre de questions en amont, pour que la procédure se déroule de la manière la plus optimale possible. Exemples des questions abordées:

- $A u$ vu du déroulement de votre traitement, quel ou quels médecins ont/auraient pu commettre une violation du devoir de diligence?

- De quels documents avez-vous besoin?

- En quoi consiste votre dommage à la santé?

- etc.

\section{Contact, formulaire de demande, informations}

Pour faciliter le dépôt d'une demande d'expertise, nous disposons d'un formulaire en deux formats différents. Le formulaire Web peut être rempli à l'écran et nous être transmis en ligne. Le formulaire PDF peut être rempli à l'écran mais doit être signé manuellement et nous être envoyé par courrier postal. Vous trouverez les deux versions du formulaire ici: www.fmh.ch $\rightarrow$ Prestations $\rightarrow$ Droit $\rightarrow$ Bureau d'expertises extrajudiciaires de la FMH. Notre site vous fournit également de nombreuses informations sur le déroulement de la procédure.

Nos collaborateurs se tiennent volontiers à votre disposition pour tout renseignement que vous souhaiteriez: Bureau d'expertises extrajudiciaires de la FMH Case postale 65 3000 Berne 15

Tél. 03135912 10, les matins de 9 à 12 heures Fax 0313591212 